OPEN ACCESS

Edited by: Xin-Ming Shen,

Mayo Clinic, United States

Reviewed by:

Yanghua Tian

First Affiliated Hospital of Anhu

Medical University, China

K. M. Hafizur Rahman,

Women Medical College, Bangladesh

Nazmul Haque,

University of Malaya, Malaysia

*Correspondence:

Yan Zhan

zhan.yan2@hotmail.com

Specialty section:

This article was submitted to Neuromuscular Disorders and

Peripheral Neuropathies,

a section of the journal

Frontiers in Neurology

Received: 17 August 2021 Accepted: 18 November 2021 Published: 15 December 2021

Citation:

Zhou J, Jiang L, Yuan S, Huang J, Shi $Q$, Xie Y, Deng B and Zhan Y

(2021) Association Study of Apolipoprotein E Gene Polymorphism

With Incidence and Delayed Resolution of Hemifacial Spasm.

Front. Neurol. 12:760126.

doi: 10.3389/fneur.2021.760126

\section{Association Study of Apolipoprotein E Gene Polymorphism With Incidence and Delayed Resolution of Hemifacial Spasm}

\author{
Jianxin Zhou, Li Jiang, Sangui Yuan, Jiashang Huang, Quanhong Shi, Yanfeng Xie, \\ Bo Deng and Yan Zhan* \\ Department of Neurosurgery, The First Affiliated Hospital of Chongqing Medical University, Chongqing, China
}

Objective: This study investigates the correlation between Apolipoprotein E gene (APOE) polymorphism and the incidence and delayed resolution of hemifacial spasms.

Methods: The APOE genotypes of 151 patients with hemifacial spasm and 73 control cases were determined by cleaved amplification polymorphism sequence-tagged sites. The distribution of three APOE alleles $(\varepsilon 2, \varepsilon 3$, and $\varepsilon 4)$ in two groups and the delayed resolution rate in 6 genotypes were calculated and statistically analyzed.

Results: The proportion of patients with APOE $\varepsilon 3 / \varepsilon 4$ genotype in the hemifacial spasm group (25.17\%) was significantly higher than that in the control group (12.33\%) $(P=0.027)$. In terms of allele frequency, the proportion of the APOE $\varepsilon 4$ allele in the hemifacial spasm group (15.56\%) was significantly higher than that in the control group (6.85\%) $(P=0.009)$. Meanwhile, the proportion of APOE $\varepsilon 4$ allele carriers in the hemifacial spasm group (29.80\%) was significantly higher than that in the control group (13.7\%) $(P=0.009)$. Logistic regression analysis showed that the $\varepsilon 4$ allele significantly increased the incidence of hemifacial spasm (OR 2.675, 95\% Cl 1.260-5.678, $P=0.010)$. Among the 32 patients with a delayed resolution, the $\varepsilon 3 / \varepsilon 3$ and $\varepsilon 3 / \varepsilon 4$ had the highest proportion in 6 genotypes. The delayed resolution rate of APOE $\varepsilon 3 / \varepsilon 4(34.21 \%)$ was significantly higher than APOE $\varepsilon 3 / \varepsilon 3$ (17.78\%) $(P<0.05)$. The delayed resolution rate of APOE $\varepsilon 4$ carriers was the highest (33.33\%) in the 3 allele carriers, but there was no significant difference among the 3 allele carriers $(P=0.065)$.

Conclusion: The polymorphism of APOE is relevant to the incidence rate of hemifacial spasms. APOE $\varepsilon 4$ allele increases the incidence of hemifacial spasm. The APOE $\varepsilon 4$ allele may promote the occurrence of delayed resolution.

Keywords: APOE, hemifacial spasm, polymorphism, delayed resolution, incidence rate

\section{INTRODUCTION}

Hemifacial spasm (HFS) refers to a common cranial nerve disease characterized by involuntary contractions of muscles, which originates from the orbicularis oculi muscle and gradually progresses to the whole facial muscle (1). At present, the etiology is still unclear. It is generally accepted that HFS results from the vascular compression of the facial nerve at the root entry zone, 
and demyelination and axonal loss are the primary pathologies (2-4). However, many people with vascular compression do not show any symptoms (5). According to the pathogenesis, the symptoms of all patients should be relieved immediately after an operation, but there are still some patients present with delayed resolution. The current theories do not fully explain the above-mentioned phenomenon. Therefore, we speculate that there might be some potential risk factors that influence myelin repair.

Apolipoprotein $\mathrm{E}$ is a major apolipoprotein that is mapped by the apolipoprotein $\mathrm{E}$ gene (APOE), which has 3 alleles (APOE $\varepsilon 2, \operatorname{APOE} \varepsilon 3$, and APOE $\varepsilon 4$ ) and 6 genotypes (6). Some studies found that APOE $\varepsilon 4$ could accelerate the pathophysiological process by interfering with remyelination $(7,8)$. Based on the fact that demyelination is a characteristic pathological change of HFS and plays an important role in the occurrence of disease, we speculate that APOE $\varepsilon 4$ may promote the occurrence of HFS and delayed resolution by affecting the repair of the myelin sheath.

In this study, we aimed to explore the association of APOE polymorphism with the incidence and delayed resolution of HFS by analyzing the differences in the distribution of APOE in different populations.

\section{DATA AND METHOD}

This retrospective case-control study included 151 patients with HFS [90 females and 61 males with an average age of 46.99 \pm 7.79 (SD) years] who were admitted to the Department of Neurosurgery, the First Affiliated Hospital of Chongqing Medical University from September 2019 to March 2021. In addition, 73 control participants [38 females and 35 males with an average age of $44.72 \pm 10.02$ (SD) years] were included in this study as the control group. The study was approved by the Bioethics Committee of the First Affiliated Hospital of Chongqing Medical University (No. 2019-258). Informed consent was obtained from all the individual participants included in the study.

\section{Inclusion and Exclusion Criteria HFS Group}

Inclusion criteria (9): consistent with the clinical manifestation of HFS, with a history longer than six months, excluded from secondary lesions by CT or MRI. Exclusion criteria: anamnesis of facial paralysis, mental illness, cerebrovascular diseases such as cerebral infarction, encephalitis, or dementia.

\section{Control Group}

Inclusion criteria: the normal controls were recruited from the healthy subjects who came to our hospital for routine physical examinations. Exclusion criteria: anamnesis of cranial nerve disease, craniocerebral trauma, intracranial space occupation, cerebrovascular disease, intracranial infection, mental illness, or dementia.

\section{APOE Genotype Identification}

A total of $2 \mathrm{~mL}$ of venous blood was collected from all participants, DNA was extracted by using Xinbaji nucleic acid
TABLE 1 | Apolipoprotein E gene (APOE) genotype decision table.

\begin{tabular}{lcc}
\hline APOE genotype & \multicolumn{2}{c}{ APOE monitoring site } \\
\cline { 2 - 3 } & $\boldsymbol{\varepsilon} 2$ & $\boldsymbol{\varepsilon} 4$ \\
\hline$\varepsilon 2 / \varepsilon 2$ & $526 \mathrm{~T} / \mathrm{T}$ & $388 \mathrm{~T} / \mathrm{T}$ \\
$\varepsilon 2 / \varepsilon 3$ & $526 \mathrm{C} / \mathrm{T}$ & $388 \mathrm{~T} / \mathrm{T}$ \\
$\varepsilon 2 / \varepsilon 4$ & $526 \mathrm{C} / \mathrm{T}$ & $388 \mathrm{~T} / \mathrm{C}$ \\
$\varepsilon 3 / \varepsilon 3$ & $526 \mathrm{C} / \mathrm{C}$ & $388 \mathrm{~T} / \mathrm{T}$ \\
$\varepsilon 3 / \varepsilon 4$ & $526 \mathrm{C} / \mathrm{C}$ & $388 \mathrm{~T} / \mathrm{C}$ \\
$\varepsilon 4 / \varepsilon 4$ & $526 \mathrm{C} / \mathrm{C}$ & $388 \mathrm{C} / \mathrm{C}$ \\
\hline
\end{tabular}

extraction reagent. The DNA of the participants, the weak positive control group, and the blank group were added to the reaction tube that had APOE gene detection reagents (provided by Wuhan YZY Biopharma); then installed into the Applied Biosystems 7,500 Real-Time PCR system for amplification (the first stage: $37^{\circ} \mathrm{C} 10 \mathrm{~min}, 1$ cycle; the second stage: $95^{\circ} \mathrm{C} 5 \mathrm{~min}$, 1 cycle; the third stage: $95^{\circ} \mathrm{C} 15 \mathrm{~s}, 60^{\circ} \mathrm{C} 60 \mathrm{~s}, 40$ cycles; signal acquisition: the third stage was collected FAM, VIC, and ROX signals under $60^{\circ} \mathrm{C}$ and enabled the Real-Time PCR). The genotype was determined according to the $\mathrm{Ct}$ value of the FAM channel and VIC channel, The genotyping method was shown in Table 1.

\section{Grading the Degree of Spasm in Patients With HFS}

The symptomatic change (SMC) grading system (Grade I: Localized spasm of the orbicularis oculi; Grade II: The spasm spreads to other ipsilateral facial muscles; Grade III: Vision is affected because of the frequent spasms; Grade IV: Disfiguring asymmetry) that developed by Kwan Park et al. (10) was used in this study to grade the degree of spasm in patients with HFS. The correlation between the degree of spasm and the course of the disease was further analyzed.

\section{Classification of Postoperative Symptoms}

The symptoms after the microvascular decompression of HFS were divided into four types (11). Type 1: The symptoms disappeared immediately after operation; Type 2: The symptoms were relieved after an operation and gradually disappeared; within 7 days to 2 years; Type 3: The symptoms disappeared immediately after the operation, but recurred within 3 days, and then gradually disappeared and Type 4: Invalid after the operation.

\section{Statistical Analysis}

All data were analyzed by SPSS v.22. Measured data were expressed as mean $\pm S D(x \pm S D)$ and tested by the $t$-test. Enumeration data were tested by Pearson chi-square test or Fisher's exact test. Risk factors analysis was conducted using the logistic regression model. All tests of significance were two-sided with a $p$-value $<0.05$. 
TABLE 2 | Comparative analysis table between the hemifacial spasm (HFS) group and the control group $(n, x \pm s)$.

\begin{tabular}{lcccc}
\hline Clinical data & $\begin{array}{c}\text { Control group } \\
(\boldsymbol{n}=\mathbf{7 3})\end{array}$ & $\begin{array}{c}\text { HFS group } \\
(\boldsymbol{n}=\mathbf{1 5 1})\end{array}$ & $\boldsymbol{t} / \chi^{2}$ value & $\boldsymbol{P}$ value \\
\hline Gender male & 35 & 61 & 1.145 & 0.285 \\
Female & 38 & 90 & & \\
Age (year) & $44.71 \pm 10.02$ & $46.99 \pm 7.79$ & 1.710 & 0.090 \\
Hypertension & 17 & 40 & 0.266 & 0.606 \\
\hline
\end{tabular}

\section{RESULTS}

\section{General Data}

A total of 151 patients with HFS were included in this study. Among them, 78 cases were left-sided, 73 cases were right-sided. The mean course of the disease was $65.88 \pm 63.99$ (SD) months (range 12-360 months). As shown in Table 2, there was no statistical difference between the two groups in gender, age, and incidence of hypertension $(P>0.05)$.

\section{The Correlation Between the Degree of Spasm and the Course of the Disease}

There were 29 patients with grade I HFS, 67 patients with grade II HFS, 46 patients with grade III HFS, and 9 patients with grade IV HFS. Based on the SMC grading system, the mean course of the disease was 47.6 months (range: $12-180$ months) in grade I, 55.3 months (range: 12-204 months) in grade II, 71.2 months (range: 7.5-240 months) in grade III, and 176 months (range: 24-360 months) in grade IV patients. We found that the longer disease duration was associated with higher SMC grade $(P<0.05)$.

\section{Genotype Distribution}

APOE has 3 alleles (APOE $\varepsilon 2$, APOE $\varepsilon 3$, and APOE $\varepsilon 4$ ) and 6 phenotypes $(\varepsilon 2 / \varepsilon 2, \varepsilon 2 / \varepsilon 3, \varepsilon 2 / \varepsilon 4, \varepsilon 3 / \varepsilon 3, \varepsilon 3 / \varepsilon 4$, and $\varepsilon 4 / \varepsilon 4)$. The genotype distribution, allele frequencies, and allele carriers frequencies of patients with HFS and the control group are shown in Table 3. In the control group, there were 1 of $\varepsilon 2 / \varepsilon 2,10$ of $\varepsilon 2 / \varepsilon 3,1$ of $\varepsilon 2 / \varepsilon 4,52$ of $\varepsilon 3 / \varepsilon 3$, and 9 of $\varepsilon 3 / \varepsilon 4$. In the HFS group, there were 2 of $\varepsilon 2 / \varepsilon 2,14$ of $\varepsilon 2 / \varepsilon 3,5$ of $\varepsilon 2 / \varepsilon 4,90$ of $\varepsilon 3 / \varepsilon 3,38$ of $\varepsilon 3 / \varepsilon 4$, and 2 of $\varepsilon 4 / \varepsilon 4$. Among 6 phenotypes, the proportion of $\varepsilon 3 / \varepsilon 4$ in the HFS group $(25.17 \%)$ was significantly higher than that in the control group $(12.33 \%)(P=0.027)$. The proportion of the APOE $\varepsilon 4$ allele carriers $(\varepsilon 2 / \varepsilon 4, \varepsilon 3 / \varepsilon 4, \varepsilon 4 / \varepsilon 4)$ in the HFS group was $29.80 \%$, which was higher than that in the control group $(13.70 \%)(P=0.009)$ (Figure 1A). The frequencies of the APOE $\varepsilon 4$ allele in the HFS group and control group were $15.56 \%$ and $6.85 \%$ (Figure 1B), the difference was also statistically significant $(P=0.009)$.

\section{Single-Factor Logistics Regression Analysis-Risk Factors of HFS}

A total of $45(29.8 \%)$ of 151 patients with HFS were APOE $\varepsilon 4$ allele carriers and $10(13.7 \%)$ of 73 control cases were $\mathrm{APOE} \varepsilon 4$ allele carriers, the difference was statistically significant $(P=0.009)$. As shown in Table 4 , logistic regression
TABLE 3 | Statistics Analysis of the HFS group and the control group ( $n$, \%).

\begin{tabular}{lccccc}
\hline & & $\begin{array}{c}\text { Control } \\
\text { group } \\
(\boldsymbol{n}=\mathbf{7 3})\end{array}$ & $\begin{array}{c}\text { HFS } \\
\text { group } \\
(\boldsymbol{n}=\mathbf{1 5 1})\end{array}$ & $\chi^{\mathbf{2}}$ value & $\boldsymbol{P}$ value \\
\hline Phenotype & $\varepsilon 2 / \varepsilon 2$ & $1(1.37)$ & $2(1.32)$ & & \\
& $\varepsilon 2 / \varepsilon 3$ & $10(13.70)$ & $14(9.27)$ & 1.008 & 0.315 \\
& $\varepsilon 2 / \varepsilon 4$ & $1(1.37)$ & $5(3.31)$ & 0.162 & 0.688 \\
& $\varepsilon 3 / \varepsilon 3$ & $52(71.23)$ & $90(59.60)$ & 2.868 & 0.090 \\
& $\varepsilon 3 / \varepsilon 4$ & $9(12.33)$ & $38(25.17)$ & 4.891 & 0.027 \\
Carrier & $\varepsilon 4 / \varepsilon 4$ & $0(0.00)$ & $2(1.32)$ & & 1.000 \\
& $\varepsilon 2$ & $12(16.44)$ & $21(13.90)$ & 0.251 & 0.616 \\
& $\varepsilon 3$ & $71(97.26)$ & 142 & 0.512 & 0.474 \\
Frequency of allele & $\varepsilon 4$ & $10(13.70)$ & $45(29.80)$ & 6.888 & 0.009 \\
& $\varepsilon 4(8.90)$ & $23(7.62)$ & 0.221 & 0.638 \\
& $\varepsilon 3$ & 123 & 232 & 3.299 & 0.069 \\
& $\varepsilon 4$ & $(84.25)$ & $(76.82)$ & & \\
& $10(6.85)$ & $47(15.56)$ & 6.729 & 0.009 \\
\hline
\end{tabular}

analysis showed that the e4 allele was found to be a significant increase in the incidence of HFS (OR 2.675, 95\%CI $1.260-5.678, P=0.010)$.

\section{Correlation Between APOE Gene and Delayed Resolution}

There were 118 cases of type 1,24 cases of type 2, 8 cases of type 3 , and 1 case of type 4 in 151 patients with HFS after the operation. One invalid patient after operation showed $\varepsilon 3 / \varepsilon 4$ genotype. There were 32 patients with delayed resolution (Type 2 and 3). Among the 32 patients, the genotype frequencies were $50.00 \%$ for $\varepsilon 3 / \varepsilon 3$, $40.62 \%$ for $\varepsilon 3 / \varepsilon 4,3.12 \%$ for $\varepsilon 2 / \varepsilon 2$, $\varepsilon 2 / \varepsilon 4$, and $\varepsilon 4 / \varepsilon 4,0 \%$ for $\varepsilon 2 / \varepsilon 3$. Because the $\varepsilon 3 / \varepsilon 3$ and $\varepsilon 3 / \varepsilon 4$ had the highest proportion in 6 genotypes, we specifically compared the delayed resolution rate of these two genotypes.

As shown in Table 5, the differences in delayed resolution rates among the 6 genotypes were statistically significant $(P=0.027)$, the delayed resolution rate of APOE $\varepsilon 3 / \varepsilon 4(34.21 \%)$ was significantly higher than APOE $\varepsilon 3 / \varepsilon 3(17.78 \%)(P<0.05)$ (Figure 2). The delayed resolution rate of APOE $\varepsilon 4$ carriers was the highest $(33.33 \%)$ in the three allele carriers, but there was no significant difference in the delayed resolution rate among the three allele carriers $(P=0.065)$.

\section{DISCUSSION}

The APOE gene consists of four exons and three introns, totaling 3,597 nucleotides. APOE has 3 alleles (APOE $\varepsilon 2$, $\mathrm{APOE} \varepsilon 3$, and APOE $\varepsilon 4)$ and 6 phenotypes $(\varepsilon 2 / \varepsilon 2, \varepsilon 2 / \varepsilon 3$, $\varepsilon 2 / \varepsilon 4, \varepsilon 3 / \varepsilon 3, \varepsilon 3 / \varepsilon 4$, and $\varepsilon 4 / \varepsilon 4)$. Among them, APOE $\varepsilon 3$ is the wild type that has normal function and the highest proportion in the population $(12,13)$. APOE $\varepsilon 2$ and $\varepsilon 4$ are mutated, $\varepsilon 4$ is recognized as a negative regulator in many diseases. Studies have also shown it can increase the risk of developing early-onset and sporadic Alzheimer's disease 


\section{A}

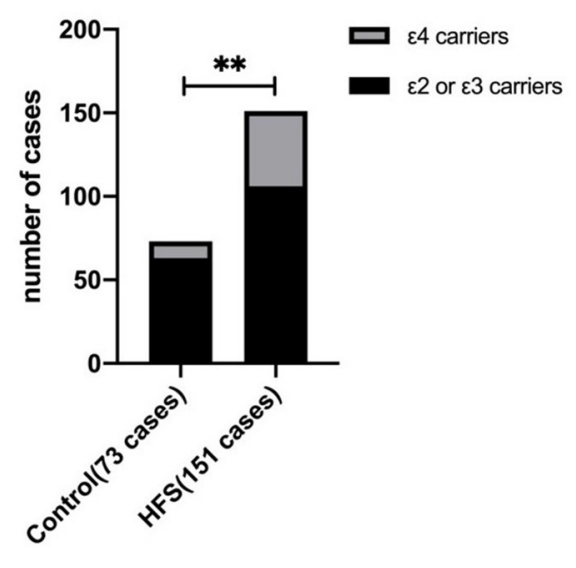

B

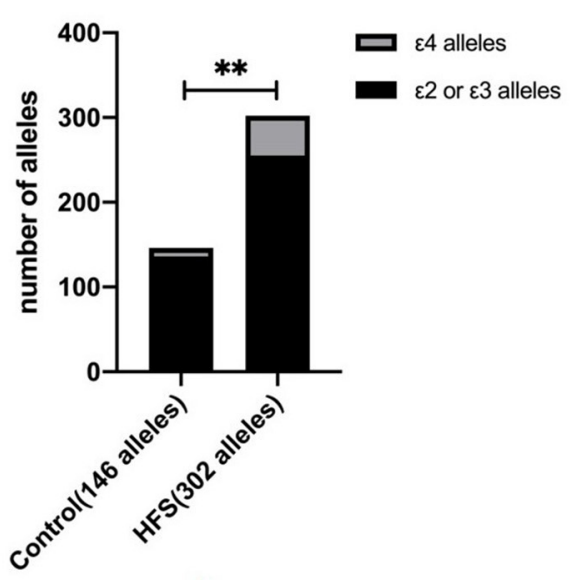

FIGURE 1 | The difference between the hemifacial spasm group and the control group in the $\varepsilon 4$ allele frequency and the $\varepsilon 4$ allele carrier. The proportion of $\varepsilon 4$ allele carriers in the hemifacial spasm group was higher than that in the control group (A). The $\varepsilon 4$ allele frequency in the hemifacial spasm group was higher than that in the control group (B). ( $\left.{ }^{\star}: P<0.001\right)$.

TABLE 4 | Single-factor logistics regression of risk factors for HFS.

\begin{tabular}{llllll}
\hline Factor & $\boldsymbol{b}$ & SE $(\boldsymbol{b})$ & Wald $\chi^{2}$ & $\boldsymbol{P}$ & OR (95\% Cl) \\
\hline$\varepsilon 2$ carrier & -0.197 & 0.394 & 0.250 & 0.617 & $0.821(0.380-1.777)$ \\
$\varepsilon 3$ carrier & -0.686 & 0.804 & 0.729 & 0.393 & $0.504(0.104-2.433)$ \\
$\varepsilon 4$ carrier & 0.984 & 0.384 & 6.560 & 0.010 & $2.675(1.260-5.678)$
\end{tabular}

(14-16). Other research considered that APOE polymorphism might affect the prognosis of traumatic brain injury patients, and the prognosis of $\varepsilon 4$ carriers is worse $(17,18)$. Besides, $\varepsilon 4$ might promote the release of inflammatory factors (19), and $\varepsilon 4$ also plays a contributory role in the pathological processes of cerebrovascular diseases and demyelinating diseases (20-23).

This study shows that the proportion of patients with APOE $\varepsilon 3 / \varepsilon 4$ genotype in the HFS group $(25.17 \%)$ is significantly higher than that in the control group $(12.33 \%)(P=0.027)$. In terms of allele, the proportion of APOE $\varepsilon 4$ allele in the HFS group $(15.56 \%)$ is significantly higher than that in the control group $(6.85 \%)(P=0.009)$. Meanwhile, the proportion of APOE \&4 allele carriers in the HFS group (29.80\%) is significantly higher than that in the control group $(13.7 \%)$ $(P=0.009)$. And the logistic regression analysis shows that the $\varepsilon 4$ allele significantly increases the incidence of HFS. Thus, we speculate that there could be a correlation between APOE polymorphism and the incidence of hemifacial spams. APOE $\varepsilon 4$ allele carriers are more likely to develop HFS compared with APOE $\varepsilon 3$ and APOE $\varepsilon 2$ allele carriers. However, the specific mechanism of APOE $\varepsilon 4$ in promoting the initiation of HFS remains unknown.

At present, the etiology and pathogenesis of HFS are still inconclusive, there are three existing theories to explain the pathogenesis. Some researchers believe that due to the lack of Schwann cells in the root exit zone of the facial nerve, long-term compression of adjacent vessels leads to a focal demyelinating lesion. The ectopic electrical activity that is transmitted across synapses occurs between the exposed axons of nerve fibers, which eventually leads to the involuntary twitch of facial muscles $(24,25)$. Other researchers believe that the facial nerve produces an antidromic conduction electrical signals due to the vascular compression in the root exit zone, it increases the excitability of the facial nerve nucleus and changes its function and structure, forming an explosive downward transmission electrical activity and causing the clinical symptoms $(26,27)$. The sympathetic theory (28-30) proposed by Chinese researchers believes that the long-term vascular compression in the root exit zone leads to the damage of the vessel wall and the demyelination of the facial nerve. The sympathetic nerve fibers that are exposed in the vessel wall are in direct contact with the exposed facial nerve, which has become a bridge between the different facial nerve fibers. When the sympathetic nerves are excited, the released neurotransmitters will act on the damaged facial nerve and make it generate action potentials. The electrical signals then spread to other branches of the facial nerve through the sympathetic nerve bridge and resulting in clinical symptoms. Therefore, HFS may be related to the demyelinating lesion, the hyperexcitability of the facial nucleus, the release of neurotransmitters, and so on.

However, many imaging and autopsy findings of posterior cranial fossa confirm that neurovascular compression also exists in the normal population (5), while not all cases develop HFS. Therefore, we suppose that there are some underlying mechanisms to promote HFS. Based on prior studies, APOE $\varepsilon 4$ was considered to affect the pathophysiological processes of several diseases by participating in multiple important signaling pathways. Some studies found that APOE $\varepsilon 4$ could accelerate 
TABLE 5 | Analysis table of delayed resolution rate in the different APOE genotypes and allele carriers (n, \%).

\begin{tabular}{|c|c|c|c|c|c|c|c|c|}
\hline Phenotype & $\varepsilon 2 / \varepsilon 2$ & $\varepsilon 2 / \varepsilon 3$ & $\varepsilon 2 / \varepsilon 4$ & $\varepsilon 3 / \varepsilon 3$ & $\varepsilon 3 / \varepsilon 4$ & $\varepsilon 4 / \varepsilon 4$ & $\chi^{2}$ value & $P$ value \\
\hline Delayed resolution & $1(50.00)$ & $0(0.00)$ & $1(20.00)$ & $16(17.78)$ & $13(34.21)$ & $1(50.00)$ & & 0.027 \\
\hline Non-delayed resolution & $1(50.00)$ & $14(100.00)$ & $4(80.00)$ & $74(82.22)$ & $25(65.79)$ & $1(50.00)$ & & \\
\hline Total & 2 & 14 & 5 & 90 & 38 & 2 & & \\
\hline Carrier & $\varepsilon 2$ & $\varepsilon 3$ & $\varepsilon 4$ & & & & $\chi^{2}$ value & $P$ value \\
\hline Delayed resolution & $2(9.52)$ & $29(20.42)$ & 15 (33.33) & & & & 5.457 & 0.065 \\
\hline Non-delayed resolution & 19 (90.48) & $113(79.58)$ & $30(66.67)$ & & & & & \\
\hline Total & 21 & 142 & 45 & & & & & \\
\hline
\end{tabular}

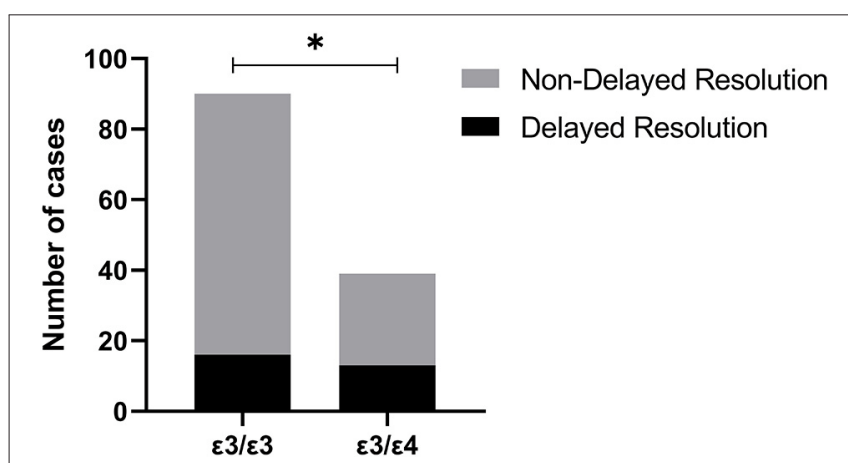

FIGURE 2 | The delayed resolution rate of Apolipoprotein E gene (APOE) $\varepsilon 3 / \varepsilon 4$ (34.21\%) was significantly higher than APOE $\varepsilon 3 / \varepsilon 3(17.78 \%)\left({ }^{*}: P<0.05\right)$.

pathophysiological processes by interfering with neurons and remyelination $(7,8)$. Pathological changes such as demyelination, axon loss, and myelin hyperplasia could be found in patients with HFS (4). We then speculate that the repair of myelin sheath in the compressed area of nerves interfered in the $\varepsilon 4$ gene carriers, thus the heterotopic conduction of electrical signal is more likely to occur. APOE $\varepsilon 4$ is possibly related to neuro-excitatory toxicity caused by the excessive release of excitatory amino acids (31). It was also found that APOE $\varepsilon 4$ has no anti-inflammatory as APOE $\varepsilon 2$ and APOE $\varepsilon 3$ do. Instead, APOE $\varepsilon 4$ promotes the secretion of pro-inflammatory and exacerbates neuroinflammation $(32,33)$. Interleukin- 6 and other inflammatory cytokines in HFS patients are found to be a higher percentage than that in normal people (34). Therefore, APOE $\varepsilon 4$ might cause HFS by promoting the release of inflammatory factors, with which patients are more prone to HFS than that with APOE $\varepsilon 2$ and APOE $\varepsilon 3$. Still, we need further theoretical basis and clinical research to corroborate the above hypothesis.

At present, the exact pathogenesis of delayed resolution after microvascular decompression is still unclear. Moller and Hatem $(35,36)$ proposed that in patients with a delayed resolution, it took some time for the recovery of demyelination changes and the high excitability of the facial nerve nucleus. Even if vascular compression has been relieved, the myelin regeneration and recovery of facial nucleus excitability still need a period. Kwan et al. (37) reported that the third month after surgery was the earliest time to predict the surgical outcome for delayed resolution patients. Due to the APOE $\varepsilon 4$ gene could affect the repair of the myelin sheath, according to the mechanism of the delayed resolution, we speculated that the incidence of demyelination changes and postoperative delayed resolution in APOE $\varepsilon 4$ carriers' population should be higher than that in APOE $\varepsilon 2$ or $\varepsilon 3$ carriers. In this study, we observed that the delayed resolution rate of $\mathrm{APOE} \varepsilon 3 / \varepsilon 4(34.21 \%)$ was significantly higher than that of APOE $\varepsilon 3 / \varepsilon 3(17.78 \%)(P<0.05)$. This finding strongly corroborates our hypothesis. The delayed resolution rate of APOE $\varepsilon 4$ carriers was the highest (33.33\%) in the three allele carriers, but the difference was not statistically significant. As a single-center study, the sample size of this research is small. A larger sample group and multi-center study are needed for further verification of the role of APOE $\varepsilon 4$ in the delayed resolution of HFS.

This study shows that the APOE $\varepsilon 4$ allele increases the incidence of HFS. We speculate that due to the APOE $\varepsilon 4$ allele promoting secretion of pro-inflammatory and exacerbating neuroinflammation, the repair of the myelin sheath at the compression area was interfered with, thus the ectopic conduction of electrical signals comes easier. And we speculate that the $\mathrm{APOE} \varepsilon 4$ allele may promote the occurrence of the delayed resolution, but larger sample size studies are needed for further verification. This study puts forward new evidence to further clarify the pathogenesis and prognosis of HFS.

\section{DATA AVAILABILITY STATEMENT}

The original contributions presented in the study are included in the article/Supplementary Material, further inquiries can be directed to the corresponding author/s.

\section{ETHICS STATEMENT}

The studies involving human participants were reviewed and approved by Bioethics Committee of the First Affiliated Hospital of Chongqing Medical University (No. 2019-258). The patients/participants provided their written informed consent to participate in this study.

\section{AUTHOR CONTRIBUTIONS}

JZ and YZ contributed to conception and designed research. SY, LJ, QS, and BD organized the database and performed 
the statistical analysis. JZ wrote the first draft of the manuscript. YZ responsible for final revision. YX, QS, $\mathrm{JH}$, and LJ wrote sections of the manuscript. All authors contributed to manuscript revision, read, and approved the submitted version.

\section{REFERENCES}

1. Mercier $\mathrm{P}$, Sindou $\mathrm{M}$. Introduction to primary hemifacial spasm: A neurosurgical disease. Neurochirurgie. (2018) 64:7981. doi: 10.1016/j.neuchi.2018.04.009

2. Jannetta PJ, Abbasy M, Maroon JC, Ramos FM, Albin MS. Etiology and definitive microsurgical treatment of hemifacial spasm. Operative techniques and results in 47 patients. J. Neurosurg. (1977) 47:3218. doi: 10.3171/jns.1977.47.3.0321

3. Mizobuchi Y, Nagahiro S, Kondo A, Arita K, Date I, Fujii Y, et al. Prospective, multicenter clinical study of microvascular decompression for hemifacial spasm. Neurosurgery. (2021) 88:846-54. doi: 10.1093/neuros/ nyaa549

4. Ruby JR, Jannetta PJ. Hemifacial spasm: ultrastructural changes in the facial nerve induced by neurovascular compression. Surg Neurol. (1975) 4:369-70.

5. Deep NL, Fletcher GP, Nelson KD, Patel AC, Barrs DM, Bendok BR, et al. Magnetic resonance imaging assessment of vascular contact of the facial nerve in the asymptomatic patient. J Neurol Surg B, Skull Base. (2016) 77:5039. doi: 10.1055/s-0036-1584196

6. Lyall DM, Ward J, Ritchie SJ, Davies G, Cullen B, Celis C, et al. Alzheimer disease genetic risk factor APOE e4 and cognitive abilities in 111,739 UK Biobank participants. Age Ageing. (2016) 45:511-7. doi: 10.1093/ageing/afw068

7. Pinholt M, Frederiksen JL, Andersen PS, Christiansen M. Apo E in multiple sclerosis and optic neuritis: the apo E-epsilon4 allele is associated with progression of multiple sclerosis. Multiple Sclerosis. (2005) 11:5115. doi: 10.1191/1352458505ms1207oa

8. Shin S, Walz KA, Archambault AS, Sim J, Bollman BP, KoenigsknechtTalboo J, et al. Apolipoprotein $\mathrm{E}$ mediation of neuro-inflammation in a murine model of multiple sclerosis. J Neuroimmunol. (2014) 271:817. doi: 10.1016/j.jneuroim.2014.03.010

9. Lefaucheur JP, Ben Daamer N, Sangla S, Le Guerinel C. Diagnosis of primary hemifacial spasm. Neurochirurgie. (2018) 64:82-6. doi: 10.1016/j.neuchi.2017.12.003

10. Lee JA, Jo KW, Kong DS, Park K. Using the new clinical grading scale for quantification of the severity of hemifacial spasm: correlations with a quality of life scale. Stereotact Funct Neurosurg. (2012) 90:169. doi: $10.1159 / 000330396$

11. Li CS. Varied patterns of postoperative course of disappearance of hemifacial spasm after microvascular decompression. Acta Neurochirurgica. (2005) 147:617-20. doi: 10.1007/s00701-005-0492-8

12. Corbo RM, Scacchi R. Apolipoprotein E (APOE) allele distribution in the world. Is APOE*4a "thrifty" allele? Ann Human Genet. (1999) 63:30110. doi: 10.1046/j.1469-1809.1999.6340301.x

13. Mahley RW, Weisgraber KH, Huang Y. Apolipoprotein E: structure determines function, from atherosclerosis to Alzheimer's disease to AIDS. J Lipid Res. (2009) 50:S183-8. doi: 10.1194/jlr.R800069JLR200

14. Davis AA, Inman CE, Wargel ZM, Dube U, Freeberg BM, Galluppi A, et al. APOE genotype regulates pathology and disease progression in synucleinopathy. Sci Transl Med. (2020) 12:529. doi: 10.1126/scitranslmed.aay3069

15. Kanekiyo T, Xu H, Bu G. ApoE and $A \beta$ in Alzheimer's disease: accidental encounters or partners? Neuron. (2014) 81:740-54. doi: 10.1016/j.neuron.2014.01.045

16. van der Kant R, Goldstein LSB, Ossenkoppele R. Amyloid- $\beta$-independent regulators of tau pathology in Alzheimer disease. Nat Rev Neurosci. (2020) 21:21-35. doi: 10.1038/s41583-019-0240-3

\section{SUPPLEMENTARY MATERIAL}

The Supplementary Material for this article can be found online at: https://www.frontiersin.org/articles/10.3389/fneur. 2021.760126/full\#supplementary-material

17. Jiang L, Yin X, Yin C, Zhou S, Dan W, Sun X. Different quantitative EEG alterations induced by TBI among patients with different APOE genotypes. Neurosci Lett. (2011) 505:160-4. doi: 10.1016/j.neulet.2011.10.011

18. Yin C, Zhou S, Jiang L, Sun X. Mechanical injured neurons stimulate astrocytes to express apolipoprotein E through ERK pathway. Neurosci Lett. (2012) 515:77-81. doi: 10.1016/j.neulet.2012.03.023

19. Sun B, Jensen NR, Chung D, Yang M, LaRue AC, Cheung HW, et al. Synergistic effects of SHP2 and PI3K pathway inhibitors in GAB2overexpressing ovarian cancer. Am J Cancer Res. (2019) 9:145-59.

20. Fazekas F, Strasser-Fuchs S, Kollegger H, Berger T, Kristoferitsch W, Schmidt $\mathrm{H}$, et al. Apolipoprotein E epsilon 4 is associated with rapid progression of multiple sclerosis. Neurology. (2001) 57:853-7. doi: 10.1212/WNL.57.5.853

21. Jin ZQ, Fan YS, Ding J, Chen M, Fan W, Zhang GJ, et al. Association of apolipoprotein E 4 polymorphism with cerebral infarction in Chinese Han population. Acta Pharmacol Sin. (2004) 25:352-6.

22. Lin B, Dan W, Jiang L, Yin XH, Wu HT, Sun XC. Association of APOE polymorphism with the change of brain function in the early stage of aneurysmal subarachnoid hemorrhage. Acta Neurochirurgica Suppl. (2011) 110:39-42. doi: 10.1007/978-3-7091-0353-1_7

23. Pinholt M, Frederiksen JL, Christiansen M. The association between apolipoprotein E and multiple sclerosis. Eur J Neurol. (2006) 13:57380. doi: 10.1111/j.1468-1331.2006.01360.x

24. Kuroki A, Møller AR. Chronic vascular irritation of the facial nerve causes facial spasm in rats. Neurol Res. (1994) 16:2848. doi: 10.1080/01616412.1994.11740241

25. Ziyal IM, Ozgen T. Microanatomy of the central myelin-peripheral myelin transition zone of the trigeminal nerve. Neurosurgery. (2007) 60:E582. doi: 10.1227/01.NEU.0000255367.19228.C4

26. Møller AR, Jannetta PJ. On the origin of synkinesis in hemifacial spasm: results of intracranial recordings. J Neurosurg. (1984) 61:56976. doi: 10.3171/jns.1984.61.3.0569

27. Yamakami I, Oka N, Higuchi Y. Hyperactivity of the facial nucleus produced by chronic electrical stimulation in rats. J Clin Neurosci. (2007) 14:45963. doi: 10.1016/j.jocn.2006.04.010

28. Zheng X, Hong W, Tang Y, Wu Z, Shang M, Zhang W, et al. Sympathetic nerves bridge the cross-transmission in hemifacial spasm. Neurosci Lett. (2012) 517:52-5. doi: 10.1016/j.neulet.2012.04.023

29. Zheng X, Hong W, Tang Y, Ying T, Wu Z, Shang M, et al. Discovery of a new waveform for intraoperative monitoring of hemifacial spasms. Acta Neurochir. (2012) 154:799-805. doi: 10.1007/s00701-012-1304-6

30. Zhou QM, Zhong J, Jiao W, Zhu J, Yang XS, Ying TT, et al. The role of autonomic nervous system in the pathophysiology of hemifacial spasm. Neurol Res. (2012) 34:643-8. doi: 10.1179/1743132812Y.00000 00057

31. Lynch JR, Tang W, Wang H, Vitek MP, Bennett ER, Sullivan PM, et al. APOE genotype and an ApoE-mimetic peptide modify the systemic and central nervous system inflammatory response. J Biol Chem. (2003) 278:4852933. doi: 10.1074/jbc.M306923200

32. Kumar A, Kumar P, Prasad M, Misra S, Kishor Pandit A, Chakravarty K. Association between Apolipoprotein $\varepsilon 4$ gene polymorphism and risk of ischemic stroke: a meta-analysis. Ann Neurosci. (2016) 23:11321. doi: $10.1159 / 000443568$

33. Zheng CZ Qi XM, Shu YB, Bai YW, Wu YG. Associations between apolipoprotein $\mathrm{E}$ gene polymorphisms and cardiovascular complications of uremic patients on maintenance hemodialysis. Blood Purif. (2018) 46:4855. doi: 10.1159/000486846

34. Chen M, Yang M, Zhou WP Li ST. Preliminary study on the relationship between inflammation and hemifacial spasm. World Neurosurg. (2019) 125:e214-e20. doi: 10.1016/j.wneu.2019.01.044 
35. Hatem J, Sindou M, Vial C. Intraoperative monitoring of facial EMG responses during microvascular decompression for hemifacial spasm. Prognost Value Long-Term Outc. (2001) 15:496-9. doi: 10.1080/026886901201 05101

36. Møller AR. Pathophysiology of hemifacial spasm. In: Hemifacial Spasm. (1994). p. S78-81. doi: 10.1007/978-3-642-85090-5_22

37. Park JS, Kong DS, Lee JA, Park K. Chronologic analysis of symptomatic change following microvascular decompression for hemifacial spasm: value for predicting midterm outcome. Neurosurg Rev. (2008) 31:4138. doi: 10.1007/s10143-008-0150-8

Conflict of Interest: The authors declare that the research was conducted in the absence of any commercial or financial relationships that could be construed as a potential conflict of interest.
Publisher's Note: All claims expressed in this article are solely those of the authors and do not necessarily represent those of their affiliated organizations, or those of the publisher, the editors and the reviewers. Any product that may be evaluated in this article, or claim that may be made by its manufacturer, is not guaranteed or endorsed by the publisher.

Copyright (c) 2021 Zhou, Jiang, Yuan, Huang, Shi, Xie, Deng and Zhan. This is an open-access article distributed under the terms of the Creative Commons Attribution License (CC BY). The use, distribution or reproduction in other forums is permitted, provided the original author(s) and the copyright owner(s) are credited and that the original publication in this journal is cited, in accordance with accepted academic practice. No use, distribution or reproduction is permitted which does not comply with these terms. 